\title{
A PRESENTATION OF THE WORK PERFORMED WITH THE BELGRADE LARGE MERIDIAN CIRCLE DURING THE PERIOD 1968-1988
}

\author{
S. Sadžakov, Z. Cvetković and M. Dačić \\ Astronomical Observatory \\ Volgina 7 \\ Yu-11050 Belgrade \\ Yugoslavia
}

ABSTRACT. The work performed with the Belgrade Large Meridian Circle and the results obtained during the last twenty years are presented.

1. Catalogue of the declinations of the latitude programme stars (KŠZ). [Sadżakov, N.S., and Saletić, P.D.: 1972, Publ. Obs. Astron. Beograd, No. 17, 1]

The Belgrade Catalogue of Latitude Stars (KŠZ) covers the celestial sphere between $+13^{\circ}$ and $+90^{\circ}$. It contains 3957 stars between magnitudes 3.0 and 9.4. Every star was observed four times on the average, in the interval 1968-1971. The mean epoch of observations is 1969.46 . The rmserror of a single observation is 0.34. A comparison with the AGK3 was made by zones and yielded an rms difference of 0 ".28.

2. Catalogue of NPZT programme stars. [Sadžakov, S., Saletic, D., and Dačić, M.: 1981, Publ. Obs. Astron. Beograd, No. 30, 1]

The catalogue of NPZT stars is obtained on the basis of the observational material compiled by use of the Meridian Circle of Belgrade Astronomical Observatory during 1973-1980. It contains 1838 stars with magnitudes $6.5 \leq \mathrm{m} \leq 8.5$. The mean epoch of observations is 1977.02 in $\alpha$ and 1978.78 in $\delta$. The rms-error of the right ascension $\varepsilon_{\alpha} \cos \delta$ is 0.030 and of declination $\varepsilon_{\delta}$ is 0.26 . Comparison with the FK4 of about 340 stars yields an error in $\alpha$ of 0.015 and in $\delta$ of 0.14 .

3. Belgrade catalogue of double stars. [Sadžakov, S., and Dačić, M.: 1989, Publ. Obs. Astron. Beograd, in press]

In the period between March 1981 and April 1987, 1576 stars were observed in Belgrade during the Double Star Programme. The measurements and the treatment of the observational material were performed by use of the relative method. Both coordinates $\alpha$ and $\delta$ were observed simultaneously, on the average every three minutes. The rms error of a single observation of double stars between $-30^{\circ}$ to $+60^{\circ}$ is $\varepsilon_{\alpha} \cos \delta= \pm 0$ s. 026 and $\varepsilon_{\delta}= \pm 0$ ". 32 .

4. A catalogue of positions of 290 stars situated in the vicinity of radio sources. [Sadżakov, S., Dačić, M., and Cvetković, Z.: 1989, Astron. J. in press] 
Simultaneously with these observations we carried out in 1982-87 observations of 290 stars from 78 parts of the sky situated in the vicinity of radio sources. A programme star was observed on the average 5.5 in $\alpha$ and 5.8 times in $\delta$. The mean observational epoch of the catalogue is 1984.60 in $\alpha$ and 1984.70 in $\delta$. The rms-error of a single observation in right ascension is $\varepsilon_{\alpha} \cos \delta=0.024$ and 0 ".30 in $\delta$. Comparison with fundamental stars yields \pm 0.009 in $\alpha$ and \pm 0 ". 13 in $\delta$.

5. A catalogue of right ascensions and declinations of FK4 stars. [Sadžakov, S., and Dačić, M.: 1989, Astron. Astrophys. Suppl. Ser. No. 77, 411]

This catalogue contains the positions of 576 FK 4 stars observed during 1981-87. The mean epoch is 1983.90 for $\alpha$ and 1983.84 for $\delta$ with mean errors for a single observation of 0.022 in $\alpha$ and 0.32 in $\delta$.

6. General catalogue of Latitude Stars (IKŠZ). [Sadžakov, S.: 1978, Publ. Obs. Astron. Beograd, No. 24, 1]

The values of declinations and proper motions in IK $\$ Z$ were derived on the basis of about 36000 star positions, the rms error of their determination ranging from 0.20 to 0"62 with the average being 0 ".35. The rms error of the determination of a single position is $\varepsilon_{8}=0.08$ and the proper motion $\varepsilon_{\mu}$ $=0.005$ with a mean epoch of 1969.44 .

7. Declinations and proper motions of the stars of the International Latitude Service on the basis of meridian catalogues from 1929-1972 (BSKŠZ1; BSKSZZ2). [Sadžakov, S., and Saletić, D.: 1975, Publ. Obs. Astron. Beograd, No. 21, 1]

The declinations in this catalogue have been obtained with an internal accuracy of $\varepsilon_{\delta}=0.023$ and the proper motions are characterized by $\varepsilon_{\mu}=0.0023$ with a mean epoch of 1954.0 . The total number of stars is 440 .

8. Investigation of systematic errors $\Delta \delta_{\alpha}$ in the latitude observations of different observatories from their comparision with the Belgrade General Catalogue of Latitude Stars and with the photographic catalogue AGK3. [Sadżakov, S.: 1979, Publ. Obs. Astron. Beograd, No. 27,1]

The systematic differences for the errors in proper motions in the AGK3 and the present catalogues are practically the same. Our analysis establishing the existence of the systematic errors of the $\Delta \delta_{\alpha}$ type in the latitude observations are in harmony with the results of many other authors. The nonuniform data handling, non-unified coordinate systems, varying number of stars, and the swift and frequent changes in the methods of processing make research in this field dificult and to some extent even unreliable. Our publication contains some recommendations for resolving these difficulties.

9. Observations of the Sun and inner planets with the Large Meridian Circle in Belgrade. [Sadżakov, S., and Dačić, M.: 1987, IAU Colloquium No. 100, Beograd, in press]

Since 1975 the Belgrade Astronomical Observatory has made regular observations of the Sun and inner planets. During the observations of the Sun, Sukharev's filter was used before 1985 and a new one from high quality glass afterwards. The errors of observation are 0.006 and 0.02 in $\alpha$ and $\delta$ for the Sun, 0.014 and 0.06 in $\alpha$ and $\delta$ for Mercury and 0.004 and 0.03 in $\alpha$ and $\delta$ for Venus. 